\title{
ANÁLISE CRÍTICA DE BULAS SOB A PERSPECTIVA DO USUÁRIO DE MEDICAMENTOS
}

\section{A CRITICAL REVIEW OF PACKAGE INSERTS FROM THE PERSPECTIVE OF DRUGS' USERS}

\author{
PAULA, Cristiane da S. ${ }^{1}$; COSTA, Camila K. ${ }^{2}$, MIGUEL, Marilis D. ${ }^{2}$; \\ ZANIN, Sandra M.W ${ }^{2}$, SPINILLO, Carla G. ${ }^{3}$ \\ REC: 07/09 AC: 08/09 \\ ${ }^{1}$ Aluna do Programa de Pós-Graduação em Ciências Farmacêuticas da UFPR \\ ${ }^{2}$ Professora do Departamento de Farmácia da UFPR \\ ${ }^{3}$ Professora do Departamento de Design da UFPR
}

\section{RESUMO:}

O uso racional de medicamentos vem sendo discutido ao longo dos anos com a finalidade de proteger a saúde da população contra o uso indiscriminado e inadequado desses produtos. Nesse contexto, as bulas representam a principal fonte de informação dos pacientes sobre os medicamentos. Em 2003, a Agência Nacional de Vigilância Sanitária publicou a resolução RDC $n^{\circ} 140$, que, dentre outras inovações, cria uma bula específica para os pacientes, cujo texto deve apresentar um maior tamanho de letra e ser de fácil compreensão. Em 2009, a $\mathrm{RDC} \mathrm{n}^{\circ} 47$ trouxe novas regras para aperfeiçoar aspectos mais críticos para o entendimento textual. Nesse trabalho, uma pesquisa foi realizada em Curitiba, Paraná, com voluntários abordados em farmácias comerciais e postos de saúde após a aquisição de um ou mais medicamentos, com o objetivo de avaliar itens como o hábito de ler bulas e as dificuldades encontradas nessa tarefa. Mais de $80 \%$ dos entrevistados lêem bulas e dentre as principais dificuldades relatam-se, primeiramente, a linguagem de difícil entendimento e, em segundo, o tamanho pequeno das letras que inviabilizam a leitura e compreensão do texto. Dentre os itens mais lidos estão os efeitos adversos, as indicações e posologia. Avaliando os resultados, concluiu-se que as dificuldades encontradas para sua leitura permanecem as mesmas que em anos anteriores à publicação da RDC $n^{\circ} 140$. Entretanto, espera-se com a RDC $n^{\circ} 47$ que essas falhas possam ser sanadas, pois são definidas nessa resolução especialmente regras para que a apresentação textual das informações seja mais clara e precisa possível para garantir o uso do medicamento da forma correta e segura.

Palavras-chave: Bulas, Legislação, Medicamentos

\section{ABSTRACT:}

The rational use of drugs has been discussed over the years in order to protect people's health against the indiscriminate and inappropriate use of these products. In this context, the package inserts represent the main source of information for patients with regards to drugs. In 2003 the National Sanitary Surveillance Agency published the resolution $n^{\circ} 140$, which, among other innovations, creates a specific package inserts for patients, whose text should display a larger font and be easy to understand. In 2009 the resolution $n^{\circ} 47$ has brought new rules to improve the most critical topics for understanding text. A research conducted in Curitiba, Paraná, was carried out with volunteers in commercial pharmacies and health clinics after purchase one or more drugs, with the aim of evaluating items such as the habit of reading package inserts and the difficulties encountered in this task. Over $80 \%$ of the volunteers reads package inserts and among the main problems reported are: first, the language difficult to understand and, second, the small size of the letters that prevents the reading and understanding of the text. Adverse effects, indications and dosage are the items most frequently readed. Evaluating the results, it was concluded that the difficulties encountered in reading remain the same as in years prior to the publication of resolution $\mathrm{n}^{\circ}$ 
140. However, it is expected to resolution $n^{\circ} .47$ that these flaws can be corrected, because it is defined in this resolution specifically rules for the textual presentation of information is more clear and precise as possible to ensure correctly and safely use of the drug.

Keywords: Package inserts, Drugs

\section{INTRODUÇÃO}

No Brasil os medicamentos foram tratados por muito tempo como produto de acesso livre nas farmácias, modelo de consumo promotor da automedicação. Entretanto, nos últimos anos muitas políticas sociais vêm sendo apresentadas para a promoção do uso racional de medicamentos como forma de proteger e recuperar a saúde, tanto individual quanto coletiva da população. Nesse contexto, a Assistência Farmacêutica se tornou parte da Política Nacional de Saúde e envolve um conjunto de ações relacionadas à pesquisa, o desenvolvimento e a produção de medicamentos e insumos, bem como sua dispensação, garantia da qualidade dos produtos e serviços, acompanhamento e avaliação da sua utilização com a expectativa de obter resultados positivos na melhoria da qualidade de vida da população (BRASIL, 2004; BRASIL, 2001).

Em 2001, o Ministério da Saúde no Brasil implementou a Política Nacional de Medicamentos, um programa que objetiva promover a melhoria das condições de assistência à saúde da população, com o propósito de garantir a segurança, eficácia e qualidade dos medicamentos, a promoção do seu uso racional e o acesso da população àqueles considerados essenciais (BRASIL, 2001). O uso racional de medicamentos compreende, dentre outros fatores, uma prescrição apropriada, dispensação em condições adequadas e consumo do medicamento nas doses indicadas (ANVISA, 2005).

$O$ uso irracional de medicamentos no mercado brasileiro parece crescer na mesma proporção do surgimento de novos medicamentos e de variações dos que já existem. O país conta com aproximadamente 30.000 apresentações diferentes de produtos registrados, incluindo os medicamentos de referência, genéricos, similares e fitoterápicos (NEVES et al., 2006).

De acordo com Silva et al. (2000), as bulas dos medicamentos são consideradas a principal fonte de informação aos pacientes para um melhor esclarecimento quanto à sua enfermidade e tratamento. Elas são consideradas secundárias em importância apenas em relação às informações fornecidas pelo médico, por meio da informação oral e escrita através do receituário (SILVA, 2000). As bulas representam, portanto, um meio de promoção para o uso racional de medicamentos (CALDEIRA, NEVES E PERINI, 2008).

Na tentativa de se evitar a automedicação, sem, no entanto, privar o paciente de conhecer todos os efeitos que o medicamento possa causar, a Agência Nacional de Vigilância Sanitária (ANVISA) publicou em 2003 a resolução RDC n 140 . Dentre outras inovações, a indústria farmacêutica passou a ser responsável pela elaboração de uma bula específica para o usuário, com termos de mais fácil compreensão e escritos em letras maiores, com o objetivo de melhorar o entendimento e alertar para os riscos da automedicação. Entretanto, na prática, notam-se ainda erros informacionais nos textos de bula publicados no país, embora uma nova resolução publicada em 2009, a $\operatorname{RDC} n^{\circ} 47$, tenha surgido com o propósito de aperfeiçoar aspectos mais críticos para

Visão Acadêmica, Curitiba, v.10, n.2, Jul. - Dez./2009 - ISSN 1518-5192 
o entendimento textual.

Vale a pena ressaltar que, de acordo com Spinillo et al (2009), dentre os aspectos passíveis de indução de erros de manipulação, de preparação e/ou estipulação de dosagem de medicamentos, podem-se listar a legibilidade do texto (tamanho do corpo tipográfico, espaçamento entre linhas, uso excessivo de caixa alta e/ou negrito, tamanho das colunas e alinhamento justificado do texto); leiturabilidade do texto (linguagem muito técnica); apresentação gráfica das informações (hierarquia tipográfica, separação das informações); apresentação de instruções visuais (legibilidade pictórica, omissão de representação de passos); e transparência do papel (SPINILLO et al, 2009; PADOVANI et al, 2008).

A resolução RDC n 47 foi publicada com novas diretrizes para a elaboração, harmonização e atualização de bulas, onde muitas questões que não estavam claras ou não foram definidas pela RDC no 140 foram elencadas.

Neste sentido, o objetivo deste trabalho foi avaliar qual o perfil das pessoas que fazem uso da bula e quais são os principais aspectos que limitam sua leitura sob seus pontos de vista.

\section{MATERIAL E MÉTODOS}

Para o presente trabalho foi realizada uma pesquisa conduzida por acadêmicos do Curso de Farmácia da Universidade Federal do Paraná (Projeto Atenção Farmacêutica na Formação do Acadêmico de Farmácia), cujo objetivo foi levantar dados referentes ao perfil das pessoas que freqüentam farmácias ou postos de saúde para adquirir medicamentos e aspectos textuais da bula quanto documento dirigido ao paciente.

Os dados da referida pesquisa foram coletados por meio de entrevistas com setenta voluntários com mais de 18 anos, abordados aleatoriamente em farmácias comerciais e postos de saúde da cidade de Curitiba, Paraná, após a aquisição de um ou mais medicamentos. Aplicou-se a esses voluntários um instrumento de avaliação estruturado e elaborado pelos autores do trabalho, abordando as seguintes questões: idade, sexo, grau de escolaridade, hábito de fazer a leitura da bula de medicamentos e se havia alguma dificuldade durante a leitura. Para aqueles que relataram não possuir o hábito de ler, solicitou-se uma justificativa.

\section{RESULTADOS}

Com base nos resultados da pesquisa, contatou-se que a maioria das pessoas que adquirem medicamento(s) nas farmácias ou postos de saúde são mulheres, entre 36 e 50 anos, com nível superior completo. O Gráfico 1 indica a proporção de homens e mulheres abordados, o Gráfico 2 apresenta a faixa etária dos entrevistados e o Gráfico 3 os relaciona quanto ao nível de escolaridade. 
Gráfico 1 - Distribuição conforme o sexo

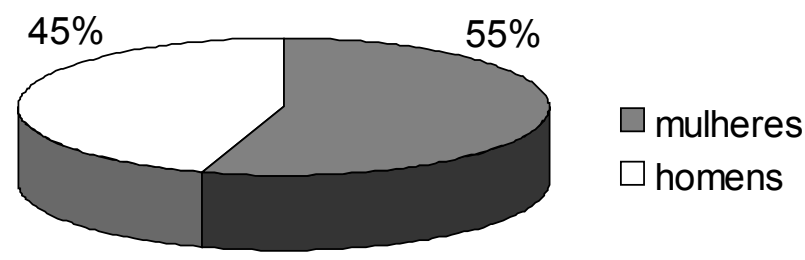

Gráfico 2 - Distribuição conforme a idade

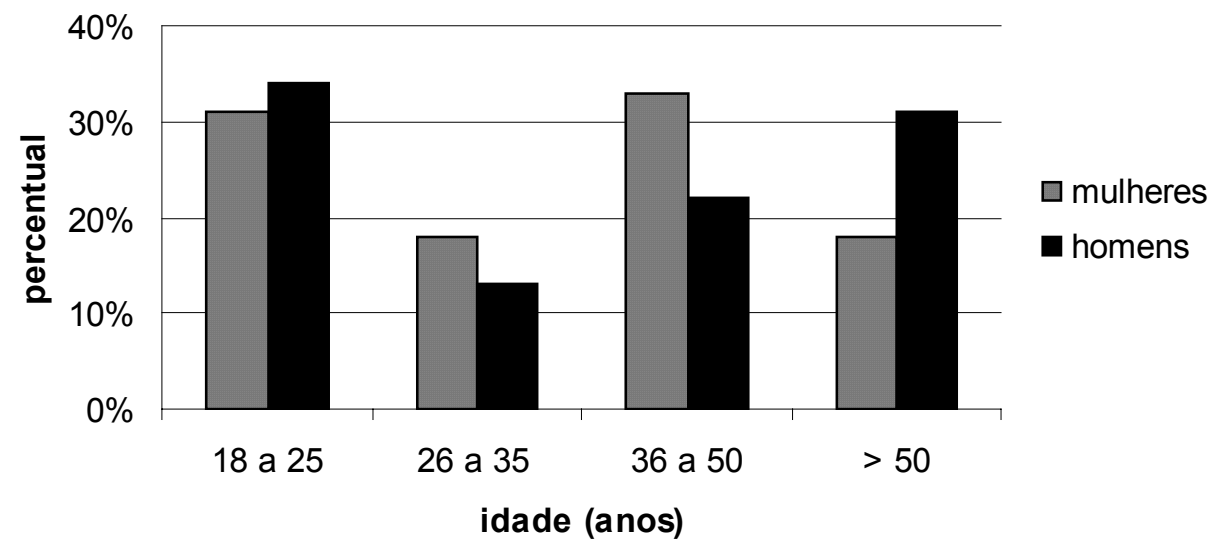

Gráfico 3 - Distribuição conforme escolaridade

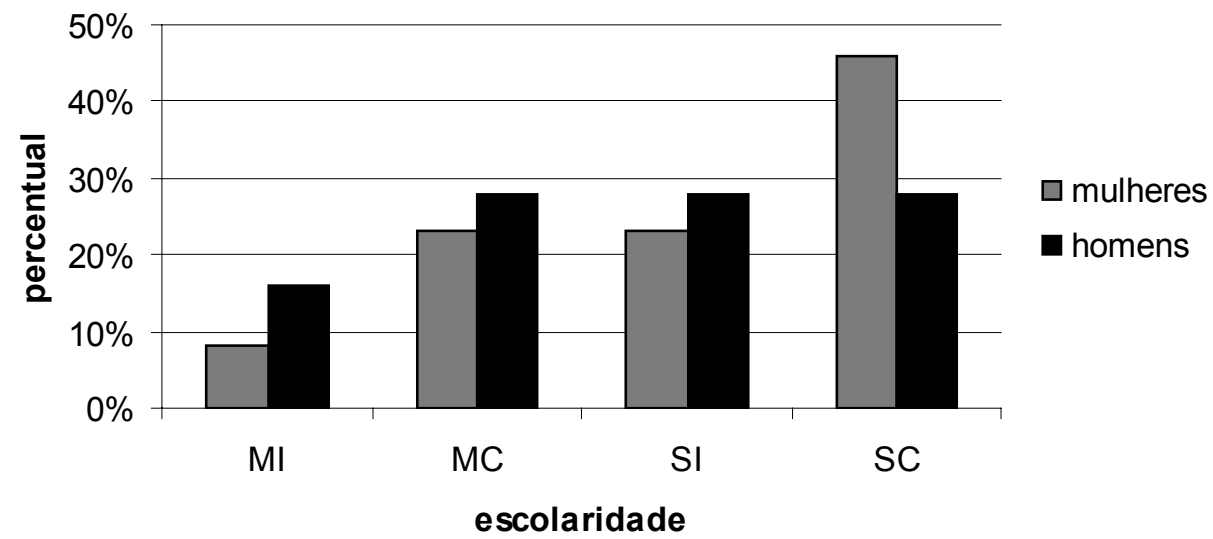

Dos entrevistados, $83 \%$ realizam a leitura de bulas de medicamentos e as principais dificuldades apontadas apresentam-se no Gráfico 4. 
Gráfico 4 - Dificuldades mais citadas na leitura da bula

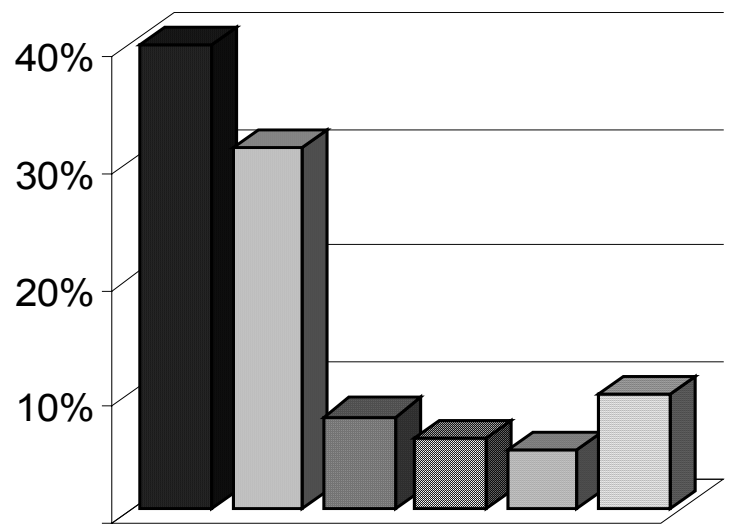

\author{
$\square$ linguagem difícil \\ $\square$ letra pequena \\ $\square$ pouco atrativa \\ $\square$ efeitos adversos como \\ influência negativa \\ $\square$ muito extensa \\ $\square$ outros
}

Mesmo não sendo realizada uma pergunta direta durante a entrevista, $6 \%$ dos entrevistados que lêem bulas expressaram o motivo de o fazerem e quais as partes que costumam ler. Dentre os que explicaram a razão de lerem bulas, $75 \%$ o fazem porque acham importante obter mais informações sobre o medicamento e $25 \%$ objetivam conferir se a prescrição está de acordo com o diagnóstico fornecido pelo médico. Os itens lidos com maior freqüência encontram-se no Gráfico 5.

Gráfico 5 - Itens lidos com maior freqüência nas bulas

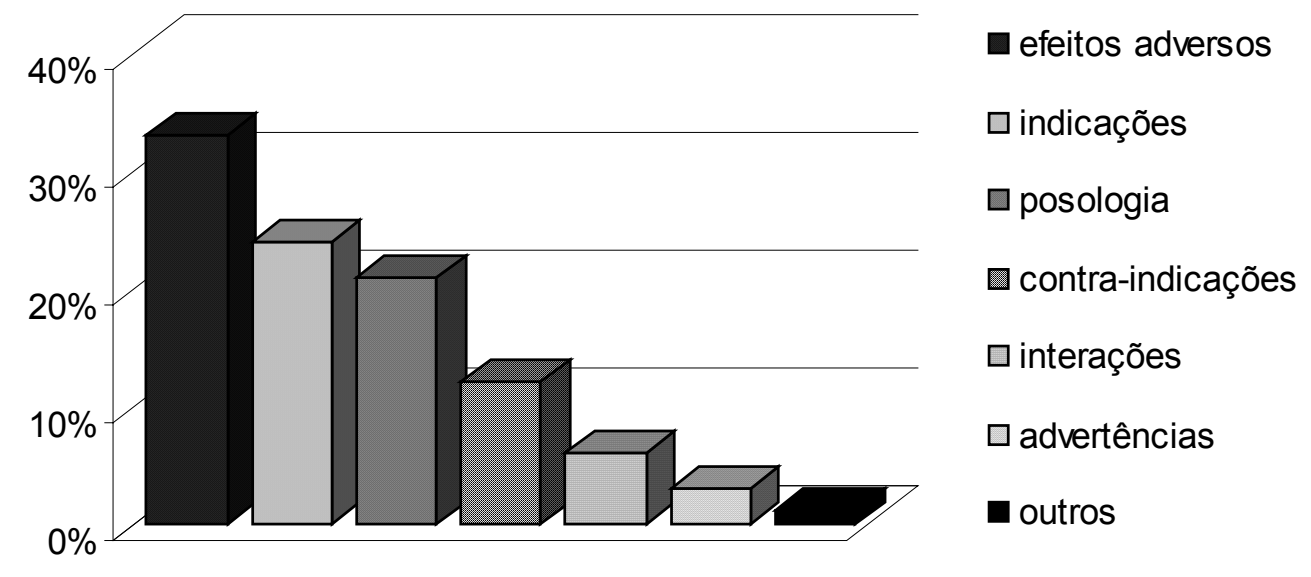

Dentre as justificativas dos $17 \%$ dos entrevistados que não realizam a leitura das bulas ao utilizar um medicamento destaca-se a fala "a explicação do médico é suficiente". Além disso, também relatam que possuem conhecimento da razão de estarem utilizando o produto e, portanto, o usuário, enquanto consumidor, não acha necessário obter mais informações. O Gráfico 6 apresenta esses resultados. 
Gráfico 6 - Justificativas dos entrevistados para não leitura das bulas

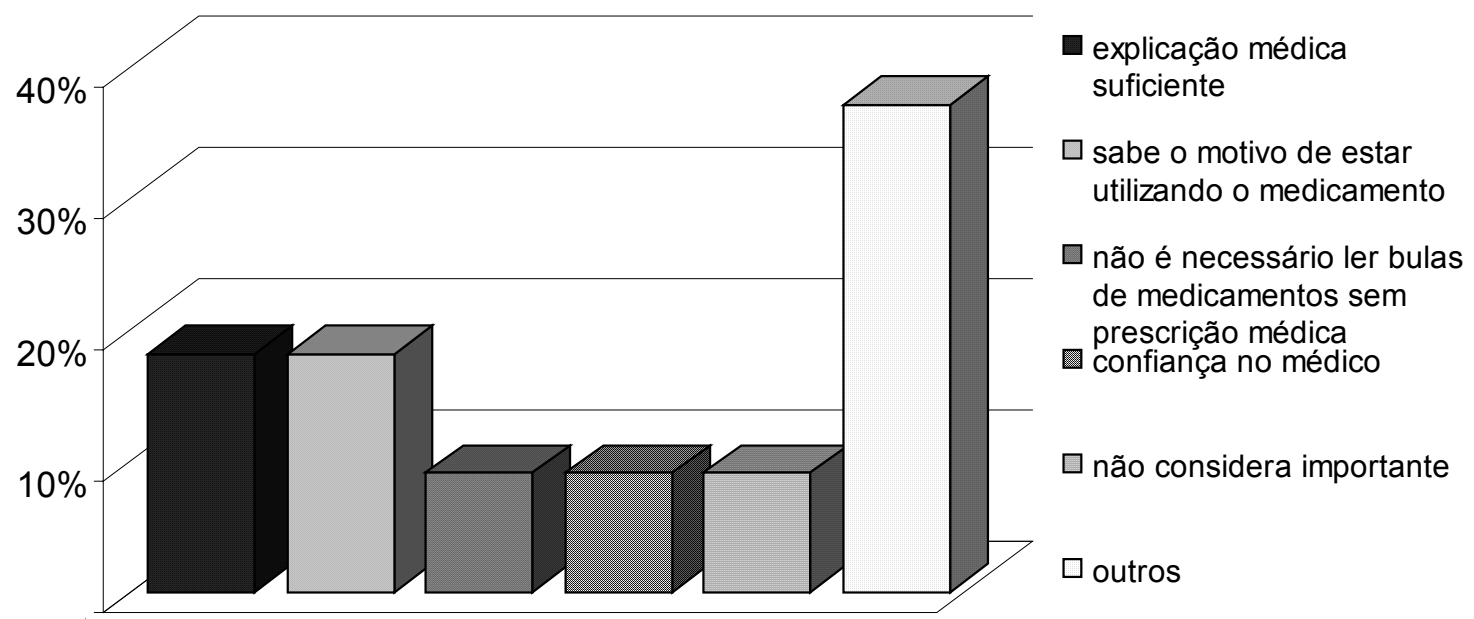

\section{DISCUSSÃO}

As legislações que tratam das bulas vêem ao longo dos anos sofrendo reestruturações para promover de forma mais eficaz a orientação correta do paciente e do profissional prescritor. Nesse contexto, a resolução RDC $n^{\circ} 140$ da ANVISA trouxe uma série de inovações das legislações anteriores, dentre elas o aumento do tamanho das letras e a criação de dois tipos de bulas, uma para o profissional da saúde e outra voltada para o paciente. Além disso, essa resolução se destacou especialmente pela criação da lista de medicamentos padrão para texto de bula, a qual objetiva harmonizar o conteúdo das bulas nacionais a partir da criação de um texto padrão para cada fármaco. Por meio desta normatização torna-se necessário também a descrição das características organolépticas a fim de facilitar a detecção de alterações de medicamentos e falsificações, a disponibilização dos textos de bula eletronicamente pelo Sistema de Gerenciamento Eletrônico de Bulas e publicação de um Compêndio de Bulas de Medicamentos.

Entretanto, uma série de pontos que não foram tratados ou não estavam claros na $R D C n^{\circ} 140$ foram regulamentados pela RDC $n^{\circ} 47$, especialmente no que se refere à apresentação textual e a determinação da presença de bula em formato especial para pessoas portadoras de deficiência visual.

Mais de $80 \%$ dos entrevistados lêem bulas e as consideram importante como fonte de informação de fácil acesso, seja para obter maior conhecimento sobre seu tratamento, seja para confirmar se a prescrição está em concordância com seu diagnóstico.

Contudo, as maiores dificuldades encontradas pelos leitores de bulas nesse trabalho são as mesmas detectadas em um estudo conduzido por Almeida et al (2006), ou seja, linguagem de difícil entendimento e o tamanho da letra muito pequeno. A relevância destes aspectos encontra-se no fato que deficiências informacionais em bulas 
podem afetar negativamente a compreensão da mensagem e acarretar o mau uso do medicamento, comprometendo o bem-estar e a saúde do paciente (WRIGHT, 1999; SLESS e TYERS, 2004; FUJITA e SPINILLO, 2006).

Vale esclarecer que há um período para a adequação das indústrias de medicamentos à RDC $n^{\circ} 47$, e por esse motivo, as bulas de medicamentos encontradas nas farmácias atualmente ainda não estão adequadas à nova resolução, principalmente no que se refere à apresentação da informação propriamente dita, pois itens como tamanho mínimo de letra de 10 pontos, fonte, espaçamentos, entrelinhas, alinhamento do texto, tamanho de colunas e aspectos textuais que diferenciem os itens e perguntas do corpo da bula, que antes não estavam definidos de forma clara pela RDC $n^{\circ} 140$, agora já estão regulamentados.

Com relação à linguagem utilizada, os textos de bula direcionados ao paciente apresentam-se na forma de perguntas e respostas simulando um diálogo com o leitor, com o objetivo de facilitar seu entendimento. No entanto, as palavras utilizadas deixam dúvidas para o leigo. Nota-se também que em muitas bulas o texto utilizado para os profissionais e para o paciente é o mesmo, sendo diferenciados somente no título dos itens, que para o paciente, vem na forma de pergunta. Além disso, são freqüentes as repetições de informações ao longo do texto. Essas deficiências devem ser sanadas pela RDC $n^{\circ} 47$, pois nela exige-se que a bula para pacientes seja clara, objetiva e possua termos explicativos após os termos técnicos, bem como não poderá mais haver a repetição de informações ao longo do texto.

A preocupação causada após a leitura dos efeitos adversos foi citada por alguns entrevistados como um problema decorrente do ato de ler bulas. Embora a RDC ${ }^{\circ}$ 140 solicite a inclusão da freqüência e gravidade dos efeitos adversos, sabe-se que em muitas bulas essas informações são insuficientes ou mal abordadas, o que pode desorientar o paciente, deixando-o desnecessariamente preocupado (SILVA et al, 2000). Com a RDC $n^{\circ} 47$, uma melhor abordagem sobre esse aspecto foi apresentada. A partir de agora, a freqüência dos efeitos adversos deve ser ordenada das mais comuns para as mais raras, utilizando alguns parâmetros para agrupá-las. Dessa forma, as reações que acontecem em $10 \%$ dos pacientes são ditas como muito comuns; se atingem de 1 a $10 \%$, são comuns; de 0,1 a $1 \%$, incomuns; $0,01 \%$ a $0,1 \%$, raras; e em menos de $0,01 \%$, muito raras. Colocando dessa maneira, os usuários-leitores conseguirão compreender mais facilmente as chances de efeitos adversos ocorrerem após o início do tratamento.

Embora menos de 10\% dos entrevistados relatem preocupação ao ler os efeitos adversos (Gráfico 4), dentre as partes da bula lidas com maior freqüência estão justamente esses efeitos, seguido das indicações e posologia (Gráfico 5). Esse resultado é diferente do encontrado por Almeida et al (2006), que em um estudo semelhante avaliou que as indicações aparecem em primeiro lugar e os efeitos adversos ficam atrás da posologia e contra indicações. Embora possa influenciar negativamente em alguns casos, a leitura dos efeitos adversos parece demonstrar a preocupação dos pacientes em conhecer as reações indesejáveis com as quais eles podem se deparar durante o tratamento. A leitura das indicações e posologia podem sugerir que o paciente tem a intenção de confirmar se a prescrição condiz com o diagnóstico e orientações médicas no que diz respeito a esses fatores. Também é possível que as explicações médicas 
durante a consulta não tenham sido claras ou o paciente não se recorde de todas as orientações, e por essa razão busca as bulas para esclarecer suas dúvidas.

Dentre aqueles entrevistados que não lêem bulas, as justificativas vão desde a confiança no médico e nas informações passadas por ele durante a consulta até explicações como de não ser necessário ler bula de medicamentos de venda livre. Embora o médico forneça as informações, as bulas nesse caso têm o objetivo de lembrá-los como utilizá-lo corretamente, uma vez que após a consulta é comum o paciente não se recordar de todas as informações recebidas, além de esclarecer as dúvidas que possam aparecer no decorrer do tratamento.

Questionamentos podem ser feitos quanto à utilização das bulas como forma de automedicação (BARROS, 1983). Acredita-se que essa preocupação justificar-se-ia caso as normatizações não tivessem evoluído nos últimos anos e ainda estivesse em vigor legislações como a Portaria $n^{\circ}$ 49/59 e o Decreto 79.094/77. Essas normas exigem a apresentação de itens como indicações, modo de usar e posologia, deixando ausente de obrigatoriedade textos como reações adversas, precauções, riscos da automedicação e conduta da superdosagem. Dessa forma, o paciente teria o conhecimento somente das informações que estimulam o uso do medicamento, mas não se referiam aos perigos de um consumo sem orientação de profissional capacitado. A partir da Portaria $n^{\circ}$ 65/84 a lista de itens obrigatórios aumentou e incorporou informações importantes sobre os riscos e cuidados no consumo de fármacos. Além disso, as RDC n 140 e 47 incorporaram ao seu texto muitas frases de alerta a serem acrescentadas nas bulas com o objetivo de chamar a atenção para os perigos da utilização de medicamentos, interrupção de tratamento ou alteração de posologia sem o conhecimento médico.

\section{CONCLUSÃO}

A bula faz parte de um abrangente sistema de informações em saúde disponível ao paciente em tratamento. De forma sintética, podemos identificar três estágios-chave no processo de tomada de informações pelo paciente em tratamento: (a) consulta com médico; (b) aquisição ou recebimento do medicamento; (c) utilização do medicamento (WAARDE, 2006). Neste último estágio, caso o medicamento esteja sendo utilizado em local fora de um centro de tratamento como clínicas ou hospitais, as informações disponíveis ao paciente se restringem ao formato escrito, sendo apresentadas na embalagem e especialmente na bula do medicamento. A fase de utilização propriamente dita do medicamento pode ser considerada a mais crítica do processo, visto que o paciente não terá acesso direto a um profissional de saúde para sanar potenciais dúvidas. Neste contexto, é fundamental que a bula apresente as informações da forma mais clara e precisa possível para garantir o uso do medicamento da forma correta e segura, o que se espera que ocorra após a reformulação das bulas baseada na RDC $n^{\circ} 47$. Cumpre ressaltar, ainda, que erros provocados por percepção ou interpretação equivocada de informações constantes nesse documento podem acarretar graves, ou mesmo irremediáveis conseqüências à saúde do paciente.

Dentro desse contexto, não se pode perder de vista as ilustrações visuais empregadas para facilitar o entendimento do modo de uso de alguns medicamentos, como por exemplo, óvulos e cremes vaginais, sprays nasais e orais, injetáveis e pós 
para reconstituição oral. A representação como forma de promover a utilização correta para uma terapêutica adequada ainda é um desafio para os profissionais formuladores de bula.

Faz-se vital, portanto, que estudos continuem sendo realizados na área a fim de identificar as causas dos problemas concernentes à leitura e compreensão da bula de medicamento pelos pacientes. Assim, medidas possam vir a ser adotadas para minimizar ou até mesmo sanar as deficiências na elaboração de bulas pela indústria farmacêutica brasileira, promovendo assim o desenvolvimento de bulas sob a perspectiva do usuário de medicamentos.

\section{REFERÊNCIAS}

ANVISA. Agência Nacional de Vigilância Sanitária. Uso racional de medicamentos: uma preocupação mundial. Disponível em: http:// www.anvisa.gov.br/DIVULGA/ noticias/2005/141005.htm. Acesso em: 03 set. 2008.

ALMEIDA, S.M.; OLIVEIRA, A.M.; CORREIA, C.C.; BENZATTI, F.P.; FERNANDES, J.T.; BARBOSA, G.R.; PIMENTA, C.P.; COSTA, T.M.M.; DONEIDA, V.C. Estudo da bula de medicamentos: uma análise da situação. Revista Brasileira de Ciências Farmacêuticas, v. 27, n. 3, p. 229-236, 2006.

BARROS, J. A. C. Estratégias mercadológicas da indústria farmacêutica e o consumo de medicamentos. Revista de Saúde Pública, v. 17, p. 377-386, 1983.

BRASIL. Secretaria Nacional de Fiscalização da Medicina e Farmácia. Portaria $\mathbf{n}^{\circ} \mathbf{4 9}$, de 10 de agosto de 1959. Regula a apresentação e o exame de rótulos e bulas de produtos farmacêuticos, produtos oficinais, dietéticos, cosméticos, de higiene, toucador.

BRASIL. Decreto $n^{\circ} 79.094$, de 5 de janeiro de 1977. Regulamenta a Lei $n^{\circ} 6.630 / 76$. BRASIL. Secretaria Nacional de Vigilância Sanitária. Portaria $n^{\circ}$ 65, de 28 de dezembro de 1984. Institui roteiro para texto de bula de medicamentos (modelo padrão de bula).

BRASIL. Ministério da Saúde. Política Nacional de Medicamentos 2001. Brasília: Ministério da Saúde, 2001. 40 p.

BRASIL. Agência Nacional de Vigilância Sanitária. Resolução de Diretoria Colegiada ${ }^{\circ}$ 140 , de 29 de maio de 2003 . Institui roteiro para texto de bula de medicamentos quanto à forma e conteúdo e publica a lista de medicamentos padrão para textos de bula.

BRASIL. Conselho Nacional de Saúde. Resolução n 338, de 6 de maio de 2004. Aprova a Política Nacional de Assistência Farmacêutica. 
BRASIL. Agência Nacional de Vigilância Sanitária. Resolução de Diretoria Colegiada $n^{\circ} 47$, de 08 de setembro de 2009. Estabelece regras para elaboração, harmonização, atualização, publicação e disponibilização de bulas de medicamentos para pacientes e para profissionais de saúde.

CALDEIRA, T.R.; NEVES, E.R.Z.; PERINI, E. Evolução histórica das bulas de medicamentos no Brasil. Cadernos de Saúde Pública, v. 24, n. 4, p. 737-743, 2008.

FUJITA, P. T. L.; SPINILLO, C. G. A apresentação gráfica de bula de medicamentos: um estudo sob a perspectiva da ergonomia informacional. In: CONGRESSO INTERNACIONAL DE ERGONOMIA E USABILIDADE 'ERGODESIGN', 2006, Bauru Anais do Congresso Internacional de Ergonomia e Usabilidade 'ERGODESIGN', 2006. Bauru: UNESP, 2006. p.1-6. 1CD-ROM. ISBN: 85-99679-02-3.

NEVES, E. R. Z. ; CALDEIRA, T.R.; PINTO, G.M.; MURASAKI, R.T. Projeto Bulas: Informação sobre medicamentos on-line. In: CONGRESSO BRASILEIRO DE INFORMÁTICA EM SAÚDE, 10., 2006, Florianópolis. Anais do $10^{\circ}$ Congresso de Informática em Saúde. São Paulo: SBIS, 2006. p. 1474-1479.

OLIVA, R. Uso racional de medicamentos: uma responsabilidade de todos. SES-SP. Secretaria de Estado da Saúde de São Paulo. Disponível em: <http://ses.sp.bvs.br/ local/File/Ricardo\%20Oliva_uso\%20racional\%20de\%20medicamentos.pdf $>$. Acesso em: 01 dez. 2008.

PADOVANI, S.; SPINILLO, C.; MIRANDA, F. Avaliação da compreensão de instruções visuais em bulas de medicamentos por usuários com baixo grau de escolaridade. In: P\&D DESIGN 2008 | $8^{\circ}$ CONGRESSO BRASILEIRO DE PESQUISA E DESENVOLVIMENTO EM DESIGN, 2008, São Paulo. Anais do 80 Congresso Brasileiro de Pesquisa e Desenvolvimento em Design. São Paulo: AEnD-BR | SENAC-SP, 2008. v. 1. p. $1098-1110$.

SILVA, T.; DAL-PIZZOL, F.; BELLO, C.M.; MENGUE, S.S.; SCHENKEL, E.P. Bulas de medicamentos e a informação adequada ao paciente. Revista de Saúde Pública, v. 34, n. 2, p. 184-189, 2000.

SLESS, D.; TYERS, A. Case history \# 5 | Panadol 24 Pack: new instructions for consumers. CRIA, 2004. Disponível em: http://www.communication.org.au/ cria_publications/ publication_id_89_1290110197.html. Acesso em 20 de outubro de 2008.

SPINILLO, C. G.; PADOVANI, S. LANZONI, C. Patient Safety: Contributions from a Task Analysis Study on Medicine Usage by Brazilians. In: Smith, M.J.; Salvendy, G. (Eds.) Human Interface and the Management of Information. Designing Information Environments vol. 5617. New York: Springer. 2009 ISBN 978-3-642-02555-6. 
WRIGHT, P. Printed Instructions: Can research make a difference? In: ZWAGA, Harm J. G. BOERSEMA, Theo. HOONHOUT, Henriëtte C. M. (Eds) Visual information for everyday use: Design and research perspectives. London, Taylor \& Francis, 1999. p. 45-66.

VAN DER WAARDE, K. Visual information about medicines for patients. In: FRASCARA, J. (Eds). Designing Effective Communications: Creating contexts for clarity and meaning. New York: Allworth Press, 2006. p. 38-50. 